\title{
LIPID-PROTEIN AND LIPID-LIPID INTERACTIONS IN CYTOCHROME OXIDASE MODEL MEMBRANES
} \author{
Patricia C. Jost $t$, Roderick A. Capadi $\$$ Garret Vanderkooi $\neq$
and O. Hayes Griffith $t$
}

\author{
tInstitute of Molecular Biology and Department of Chemistry, University of Oregon, \\ Eugene, Oregon 97403
}

$\$$ Institute for Enzyme Research, University of Wisconsin, Madison, Wisconsin 53706

\begin{abstract}
Two lipid environments are detected in membranous cytochrome oxidase, using spin labeling techniques. This model membrane system consists of closed vesicular membranes formed spontaneously when the membrane protein is isolated with its accompanying phospholipids. The data show both an immobilized component, which is constant in amount, and a fluid component. Based on spectral analysis, the interpretation is that the bound component represents a single layer of lipid immobilized on the surface of the protein between the hydrophobic protein complex and the adjacent fluid bilayer regions. Maximal enzyme activity of this functional protein complex is attained when all of the bound sites are occupied, and above this level additional phospholipid (bilayer) has little or no ef fect on the enzyme activity.
\end{abstract}

Studying lipid-protein and lipid-lipid interactions in biological membranes is difficult, partly because of the heterogeneity of the membrane proteins. The problem can be somewhat simplified by isolating from the membranes an intrinsic (i.e., integral) protein complex with its accompanying phospholipids. Cytochrome oxidase, isolated from beef heart mitochondria, is a good candidate for this approach.

Cytochrome oxidase (cytochrome $\mathrm{aa}_{3}, \mathrm{EC} 1.9 .3 .1$ ) is the terminal member of the mitochondrial electron transport chain (net chemical reaction $4 \mathrm{H}^{+}+\mathrm{O}_{2}+4 \mathrm{e}^{-} \rightarrow 2 \mathrm{H}_{2} \mathrm{O}$ ). When prepared by a variety of methods, including that of Sun et al. (1), it is a protein complex with a molecular weight of roughly $210,000(2,3)$, containing six different polypeptides of molecular weights ranging from 10,000 to $36,000(4,5)$. There are two hemes and two copper atoms in the enzymic complex (6). These moieties are most likely bound to components of 11,500 and 14,000 molecular weight (7). The overall size of the protein complex has been estimated from electron microscopy (2), and the cytochrome oxidase complex evidently spans the membrane as it can be labeled with antibodies or with chemical reagents from both the matrix and intercristal sides of the mitochondrial membrane $(8,9)$.

In cytochrome oxidase preparations lipids are isolated along with the enzymatically active protein complex, and closed membranous vesicles form spontaneously (Fig. 1) (10). These vesicles are not segments of the inner mitochondrial membrane but instead are a reasonably homogenous membrane model system. This model membrane almost 

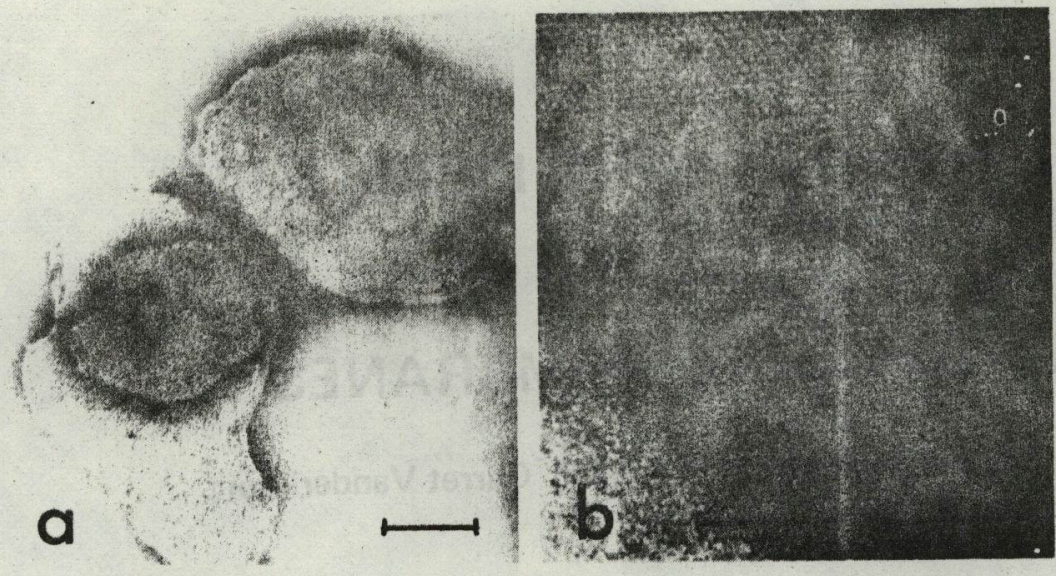

Fig. 1. Electron micrographs of membranous cytochrome oxidase $(0.49 \mathrm{mg}$ phospholipid $/ \mathrm{mg}$ protein negatively stained with $1 \%$ phosphotungstic acid, $\mathrm{pH} 5$ ). (a) Closed membranous vesicles typically seen in preparations that contain greater than $0.3 \mathrm{mg}$ phospholipid/mg protein; (b) high magnification micrograph of a crystalline region. Crystalline regions are frequently observed in vesicular preparations with 0.3 to $0.5 \mathrm{mg}$ phospholipid $/ \mathrm{mg}$ protein, in fields where several vesicles are stacked (2). Bar indicates $0.1 \mu$ : a) $50,000 x$; b) $154,000 x$. Electron micrographs by William Colguhoun.

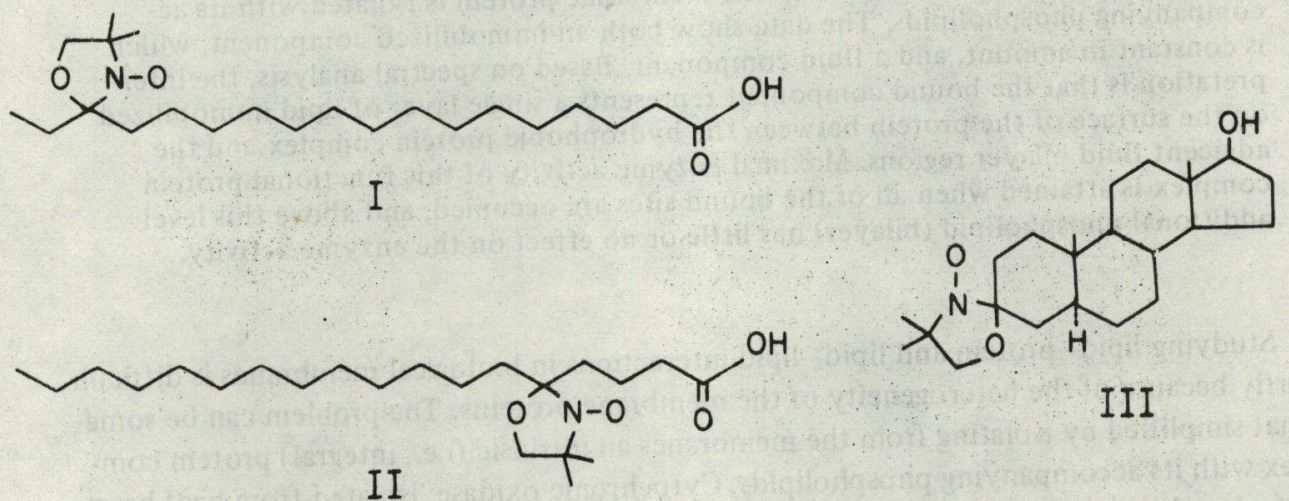

Fig. 2. Lipid spin labels that were diffused into membranous cytochrome oxidase and lipid vesicles. I, 16-doxylstearic acid: II, 5-doxylstearic acid; III, 3-doxyl-5 $\alpha$-androstan-17 $\beta$-ol, where doxyl is the trivial name for the $4^{\prime}, 4^{\prime}$-dimethyloxazolidine-N-oxyl derivative of the parent ketone (19).

certainly retains the significant lipid-protein interactions that occur in the intact inner mitochondrial membrane.

As it is usually isolated, membranous cytochrome oxidase is approximately 25 to $35 \%$ phospholipid (mg phospholipid to $\mathrm{mg}$ protein ratios of 0.33 to 0.54 ). These preparations can be lipid depleted (or lipid enriched) by established procedures (11). Recently, we have used this system and electron spin resonance spectra of fatty acid spin labels I and II (Fig. 2) diffused into these membranes to examine the properties of the protein-lipid boundary and the effect of the lipid-protein complex on lipid-lipid interactions $(12,13)$. The purpose of this paper is to review this work briefly and to present additional data relevant to the molecular properties at the protein-lipid interface. 


\section{Evidence for Boundary Lipid: Lipid-Protein Interactions}

Membranous cytochrome oxidase was isolated from beef heart mitochondria (12) and a graded series of lipid-depleted samples were prepared. (The phospholipid content ranged from 0.10 to $0.49 \mathrm{mg}$ phospholipid/mg protein.) The lipid spin labels were diffused into these buffered aqueous suspensions of cytochrome oxidase by brief mixing. Representative data obtained with the 16-doxylstearic acid spin label (I) are summarized in the left column of Fig. 3. The spectra are arranged in order of increasing lipid content of the samples, as indicated at the far left.

\section{EXPERIMENTAL SPECTRA}

\section{SUMMED SPECTRA}

$\mathrm{mg} P \mathrm{~L}$
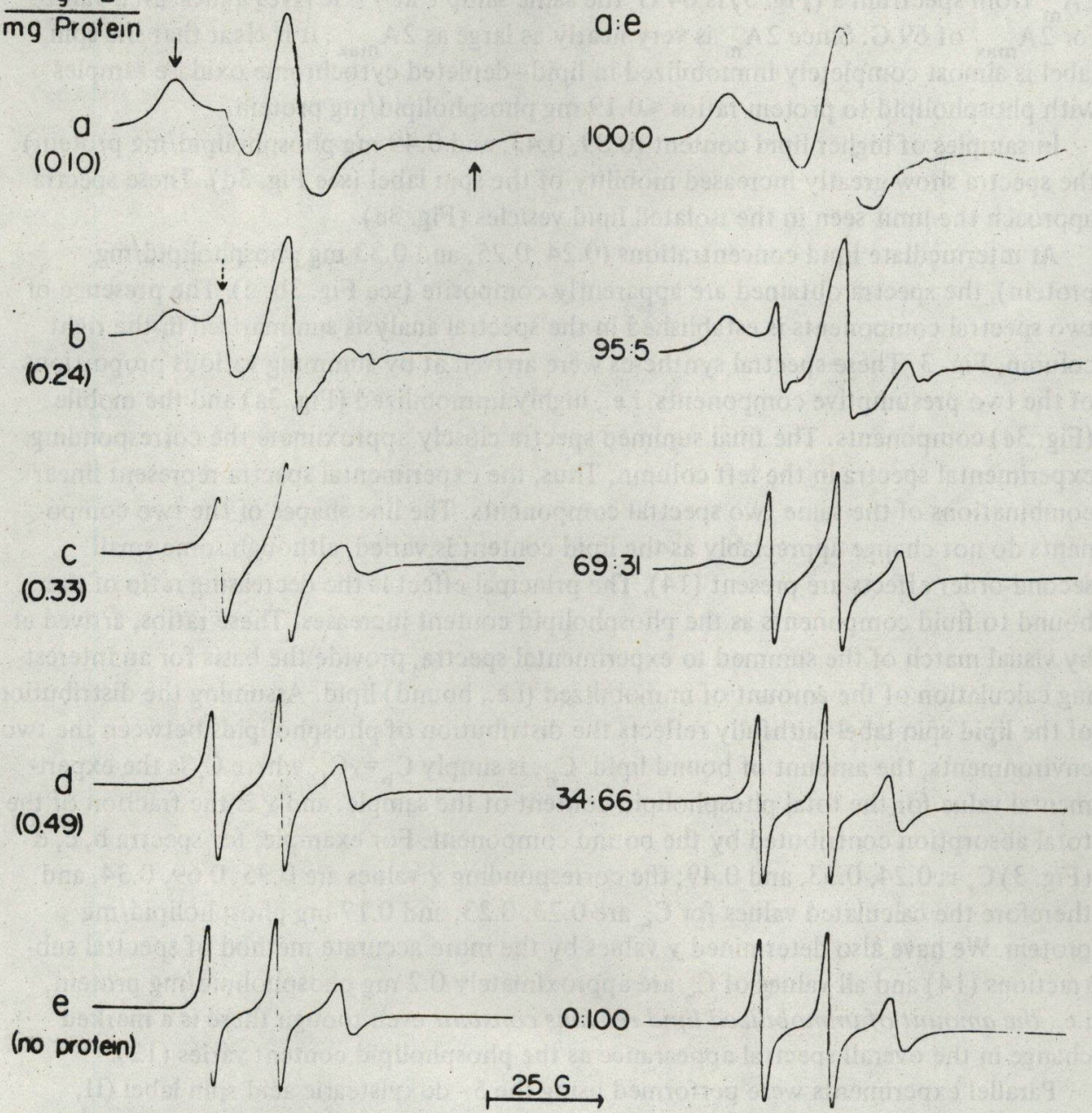

Fig. 3. Electron spin resonance spectra (left column) of 16-doxylstearic acid (I) diffused into aqueous suspensions of cytochrome oxidase with varying phospholipid content. The phuspholipid to protein ratio of each sample is indicated at the far left. Labeling was kept constant at $25 \mathrm{nmol} / \mathrm{mg}$ protein. In the right column are synthesized spectra obtained by summing various amounts of spectrum a (lipid/protein ratio of 0.10 ) and spectrum e (lipids extracted from membranous cytochrome oxidase) in the proportions indicated. All spectra have been normalized to the same center line height. Note the general similarity between the experimental and corresponding summed spectra. (Adapted from ref. 12.). 
The samples are randomly oriented so that the changes in the esr line shapes must be due to an overall increase in motion of the spin label as the lipid content of the sample increases. In general, spectral changes can be brought about by a gradual change in one spectral component or by the presence of two or more distinct components present in different proportions.

Samples at low lipid levels $(0.10,0.15,0.19 \mathrm{mg}$ phospholipid $/ \mathrm{mg}$ protein $)$ all exhibit similar line shapes that are characteristic of a highly immobilized spin label (see Fig. 3a), establishing one extreme limit in the series. The distance between the outermost lines (solid arrows, Fig. 3a) is operationally defined as $2 \mathrm{~A}_{\mathrm{m}}$, and this measurement serves as a rough indicator of molecular motion. As molecular motion decreases, $2 \mathrm{~A}_{\mathrm{m}}$ increases and eventually approaches the rigid matrix limit, $2 \mathrm{~A}_{\max }$ (or $2 \mathrm{~A}_{\mathrm{zz}}$ ). The measured value for $2 \mathrm{~A}_{\mathrm{m}}$ from spectrum a (Fig. 3 ) is $64 \mathrm{G}$; the same sample at $77^{\circ} \mathrm{K}$ gives a measured value for $2 A_{\max }$ of $69 \mathrm{G}$. Since $2 A_{m}$ is very nearly as large as $2 A_{\max }$, it is clear that the spin label is almost completely immobilized in lipid-depleted cytochrome oxidase samples with phospholipid to protein ratios $\leqslant 0.19 \mathrm{mg}$ phospholipid/mg protein.

In samples of higher lipid content $(0.39,0.43$, and $0.49 \mathrm{mg}$ phospholipid $/ \mathrm{mg}$ protein $)$ the spectra show greatly increased mobility of the spin label (see Fig. 3d). These spectra approach the limit seen in the isolated lipid vesicles (Fig. 3e).

At intermediate lipid concentrations $(0.24,0.25$, and $0.33 \mathrm{mg}$ phospholipid $/ \mathrm{mg}$ protein), the spectra obtained are apparently composite (see Fig. $3 b, c)$. The presence of two spectral components is established in the spectral analysis summarized in the right column, Fig. 3. These spectral syntheses were arrived at by summing various proportions of the two presumptive components, i.e., highly immobilized (Fig. 3a) and the mobile (Fig. 3e) components. The final summed spectra closely approximate the corresponding experimental spectra in the left column. Thus, the experimental spectra represent linear combinations of the same two spectral components. The line shapes of the two components do not change appreciably as the lipid content is varied, although some small second-order effects are present (14). The principal effect is the decreasing ratio of the bound to fluid components as the phospholipid content increases. These ratios, arrived at by visual match of the summed to experimental spectra, provide the basis for an interesting calculation of the amount of immobilized (i.e., bound) lipid. Assuming the distribution of the lipid spin label faithfully reflects the distribution of phospholipids between the two environments, the amount of bound lipid, $C_{b}$, is simply $C_{b}=\chi C_{t}$, where $C_{t}$ is the experimental value for the total phospholipid content of the sample, and $\chi$ is the fraction of the total absorption contributed by the bound component. For example, for spectra b, c, d (Fig. 3) $\mathrm{C}_{\mathrm{t}}$ is $0.24,0.33$, and 0.49 ; the corresponding $\chi$ values are $0.95,0.69,0.34$, and therefore the calculated values for $\mathrm{C}_{\mathrm{b}}$ are $0.23,0.23$, and $0.17 \mathrm{mg}$ phospholipid $/ \mathrm{mg}$ protein. We have also determined $\chi$ values by the more accurate method of spectral subtractions (14) and all values of $\mathrm{C}_{\mathrm{b}}$ are approximately $0.2 \mathrm{mg}$ phospholipid $/ \mathrm{mg}$ protein, i.e., the amount of immobilized lipid remains constant even though there is a marked change in the overall spectral appearance as the phospholipid content varies (12).

Parallel experiments were performed using the 5-doxylstearic acid spin label (II, Fig. 2) (13). With this label, the nitroxide moiety is near the polar surface of the bilayer and the two spectral components are not easily distinguished without recourse to spectral analysis. The results are consistent with those discussed above, although the uncertainties are larger because of the difficulties in judging endpoints in the spectral titrations (13).

In molecular terms, the bound lipid can be visualized as being immobilized at the lipid-protein interface. The dimensions of the cytochrome oxidase complex have pre- 
viously been estimated by electron microscopy, using the crystalline regions frequently seen in stacked vesicles (see Fig. Ib). Using these dimensions (2) and the cross-sectional areas of closely packed lipid chains from X-ray diffraction experiments (15), the amount of lipid that can be accomodated around the periphery of the protein complex is estimated to be approximately $0.2 \mathrm{mg}$ phospholipid/mg protein. This number is very close to the amount experimentally determined as bound lipid, and we refer to this component as boundary lipid (12).

The observation that this interfacial layer is immobilized is plausible, since the protein surface is irregular. The hydrophobic side groups of the polypeptide chains form the potential wells responsible for immobilization of the lipid spin labels. A similar immobilization would occur if the lipids were completely buried within the proteins, as in the Benson model (16). While we cannot completely exclude this interpretation, the electron microscopic evidence, the exchange of spin labels between the two environments (Fig. 3), and the close agreement between the observed and calculated amounts of bound lipid all argue for the boundary lipid model.

There is a small amount of phospholipid, $(0.05 \mathrm{mg}$ phospholipid/mg protein, largely cardiolipin) that cannot be removed from cytochrome oxidase without irreversibly denaturing the proteins (17). Some of this lipid may indeed be buried within the proteins, but in any case it does not appreciably affect the calculations or the conclusion that the bound lipid observed in the spin labeling experiments can best be interpreted as arising from a single boundary layer surrounding the protein complex in the plane of the membrane.

\section{Evidence for Fluid Bilayer: Lipid-Lipid Interactions}

Spin labeling provides three criteria for the presence of fluid bilayers: first, the observation of anisotropy of lipid spin labels in macroscopically oriented lipid samples (18); second, the presence of a flexibility gradient $(19,20)$ with molecular motion increasing toward the hydrocarbon end of the fatty acid spin labels; and third, the characteristic response to changes in hydration (19).

We have used these three criteria to establish the presence of phospholipid bilayers in membranous cytochrome oxidase (13). First, an example of the spectral anisotropy observed is shown in Fig. 4. At low lipid levels the samples give isotropic spectra (fig. 4a), whereas at higher lipid levels, spectral anisotropy is clearly evident (Fig. 4b). The direction

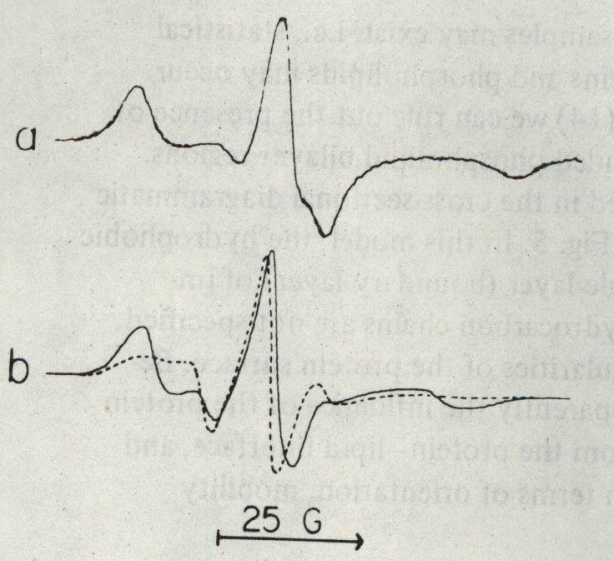

Fig. 4. Electron spin resonance spectra of cytochrome oxidase samples mounted on a glass slide and equilibrated at $100 \%$ relative humidity in a sealed chamber. The spectra were recorded with the magnetic field parallel (solid line) and perpendicular (dashed line) to the normal of the slide. The phospholipid content of the samples is (a) 0.10 and (b) $0.49 \mathrm{mg}$ phospholipid/mg protein. The two spectral components of (a) are superimposable, whereas in (b) marked anisotropy is observed. 


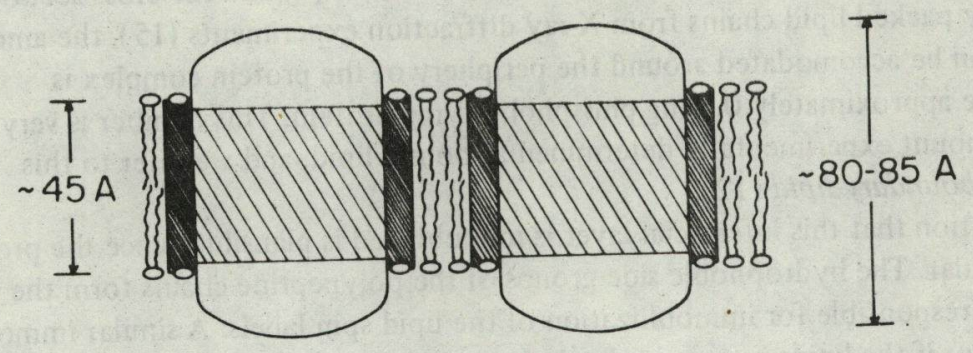

Fig. 5. Diagrammatic cross-sectional view of membranous cy tochrome oxidase. The densely crosshatched regions represent immobilized boundary lipid surrounding the hydrophobic surfaces (lightly cross-hatched) of the protein complexes. Beyond the boundary layer are the limited regions of fluid phospholipid bilayer. The hydrophylic protein surfaces and phospholipid head groups are shown exposed to the aqueous environment on both sides of the membrane. From ref. 13.

of maximum splitting is consistent with the bilayer model, and a detailed analysis of the various components of these spectra shows a remarkable similarity between the oriented component and the corresponding oriented component of the isolated lipid fraction (13).

Second, the motion in membranous cytochrome oxidase (i.e., higher lipid content) increases toward the center of the bilayer as sensed by the doxylstearic acids labeled at the 5,12 , and 16 positions. The spectral components, when analyzed, closely resemble those of the isolated lipids, which almost certainly exist as classical bilayers (13).

Third, the motion of 5-doxylstearic acid spin label in membranous cytochrome oxidase ( $0.49 \mathrm{mg}$ phospholipid/mg protein) decreases as the sample is dehydrated, and this response is similar to that seen for the isolated lipids. In contrast, the motion of the bound lipid is al ready severely restricted and does not change appreciably with changes in hydration (13). Therefore, by all three criteria, the presence of classical fluid bilayer is confirmed in membranous cytochrome oxidase vesicles. Samples with lipid content below $0.2 \mathrm{mg}$ phospholipid/mg protein possess only boundary lipid, whereas at higher lipid content both boundary lipid and fluid bilayer are present. Phospholipid in excess of the bound lipid forms fluid bilayers.

It is worthwhile noting that heterogeneity in the samples may exist, i.e., statistical fluctuations in the microscopic distribution of proteins and phospholipids may occur. However, by electron microscopy and spin labeling (14) we can rule out the presence of separate populations of protein aggregates and extended phospholipid bilayer regions.

These data and the interpretations are summarized in the cross-sectional diagrammatic view of membranous cytochrome oxidase shown in Fig. 5. In this model, the hydrophobic surfaces of the protein complex are coated by a single layer (buund.ry layer) of immobilized lipid. The detailed arrangements of the hydrocarbon chains are not specified, but they are assumed to be randomized by the irregularities of the protein surface. Beyond the boundary lipid exists the fluid bilayer. Apparently the influence of the protein on lipid structure decreases rapidly with distance from the protein-lipid interface, and the limited fluid bilayer regions behave classically in terms of orientation, mobility gradient, and response to hydration. 


\section{A Crucial Assumption}

These data clearly indicate that the spin labels detect two lipid environments, one bound and the other resembling fluid bilayer. We have shown that the number of bound sites remain essentially constant regardless of the extent of the lipid bilayer. Neither of these conclusions rest on the assumption that the distribution of the lipid spin label faithfully reflects the distribution of phospholipids in the two enviroments. However, the derivation of the $0.2 \mathrm{mg}$ phospholipid/mg protein does depend on this assumption. This crucial assumption must be tested by the use of other lipid spin labels having, if possible, different modes of anisotropic molecular motion. We have recently collected some new spin-labeling data, using the 3-doxyl-5 $\alpha$-androstan-17 $\beta$-ol spin label (III, Fig. 2), which relates to this question.

This steroid spin label (21), diffused into the same series of cytochrome oxidase preparations with varying lipid content, ${ }^{*}$ gives the spectra of Fig. 6 . Once again, the spectra from samples of low lipid content indicate that the spin labels are largely immobilized (Fig. 6a). As the lipid content increases, a more mobile component appears (Fig. 6b-d) and the spectra are clearly composite. The spectrum at high lipid content approaches the line shape observed in fluid bilayers (Fig. 6e).

The motion responsible for these changes is qualitatively different from that of the fatty acid spin labels. As discussed previously, the motion of the fatty acid spin label in the bilayer can be described as a restricted random walk, increasing in amplitude toward the center of the bilayer (19).

In contrast, the principal motion of the rigid steroid spin label III is anisotropic motion about the long axis of the molecule, as illustrated in Fig. 7. When the amplitude, $\alpha$, of motion about this axis is zero, a rigid matrix esr line shape is predicted (Fig. 7, top spectrum). At the other extreme, when $\alpha=90^{\circ}$ (rapid large amplitude motion about the $\mathrm{y}$ axis), the outermost lines move in and the spectrum sharpens, as shown in the bottom spectrum of Fig. 7. This rapid y axis molecular motion has been observed previously in model systems $(18,22,23)$ and in biological membranes (24).

In Fig. 6 (spectra $b-d)$ the composite nature is primarily due to two populations of steroid spin labels approaching the two limits of $\alpha=0^{\circ}$ (bound lipid) and $\alpha=90^{\circ}$ (fluid bilayer). The amount of bound lipid present, calculated by spectral subtraction from the data of Fig. 6 is roughly $0.2 \mathrm{mg}$ phospholipid/mg protein, in general agreement with the results obtained previously using the fatty acid spin labels.

The fraction of fluid bilayer is readily calculated once the amount of bound lipid has been determined. The results of four sets of calculations with two different spin labels are summarized in Fig.8. The values obtained using the steroid spin label are shown as squares; the other values are calculated from the earlier experiments with the 16doxylstearic acid spin label (see Table 1 of ref. 14). The agreement is surprisingly good, considering the difference in geometries and polar head groups of the two types of labels, as well as the approximations in the spectral analyses. With either spin label and with various spectral treatments, it is found that the amount of bound lipid is about $0.2 \mathrm{mg}$ phospholipid/mg protein. At lower lipid to protein ratios, no fluid bilayer is present. But once the bound sites are fully occupied, fluid bilayer regions are formed and account for

*The 3-doxyl-5 $\alpha$-androstan-17 $\beta-01$ is relatively water soluble and can be readily diffused into membranes. In contrast another common steroid spin label, 3 -doxyl-5 $\alpha$-cholestane, is very much less water soluble. It will readily incorporate into phospholipid vesicles but not into the cytochrome oxidase preparations under the conditions used here (14). 


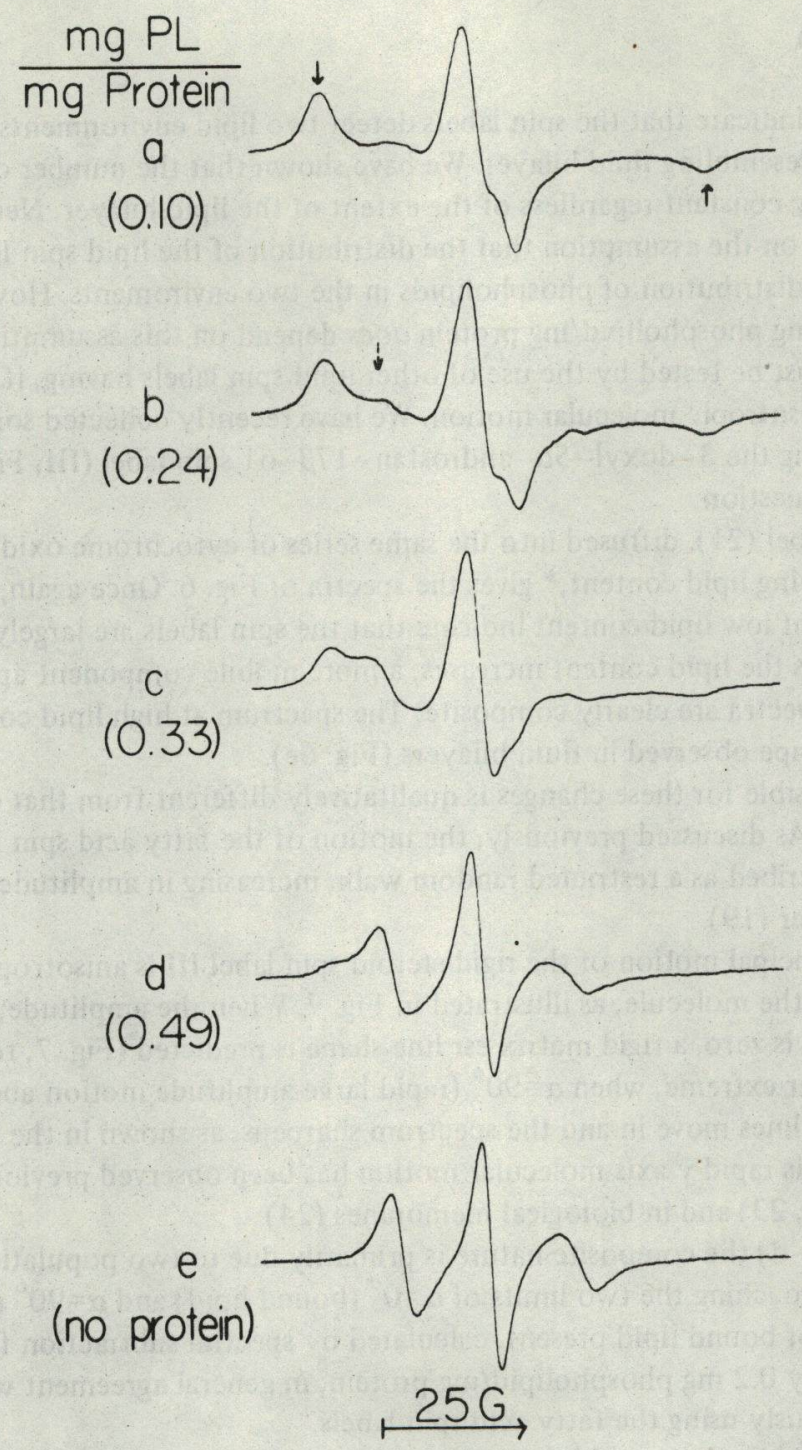

Fig. 6. Electron spin resonance spectra of $3-$ doxyl-5 $\alpha$-landrostan $-17-\beta-01$ diffused into aqueous suspensions of cytochrome oxidase with varying phospholipid content (a parallel series to Fig. 2). The solid arrows in spectrum (a) mark the positions of the outermost lines; the wide separation of these lines is characteristic of highly immobilized spin labels. In spectrum (b) the dashed arrow marks the appearance of a second, more mobile, spectral component.

an appreciable percentage of the total phospholipid at the higher phospholipid to protein ratios (Fig. 8).

\section{Correlating Biochemical and Biophysical Data on Membranous Cytochrome Oxidase}

There are a number of other experiments that relate to bound lipid in membranous cytochrome oxidase.

Two of these, discussed earlier (14), are electron microscopy data and lipid extraction 


\section{p. 276 cont.}

experiments. The morphology of the cytochrome oxidase is dependent on phospholipid content. The vesicular closed membranes are observed only in preparations with some bllayer present (i.e., slightly above $0.2 \mathrm{mg}$ phospholipid/mg protein) $(10,14,25)$. At lower phospholipid content, only amorphous reglons are seen.

The lipid extraction experiments indicate that a proportion of the phospholipid is readily extractable with cold aqueous acetone, but $0.2 \mathrm{mg}$ phosphollpid/mg protein is considerably more resistant to extraction (14). 


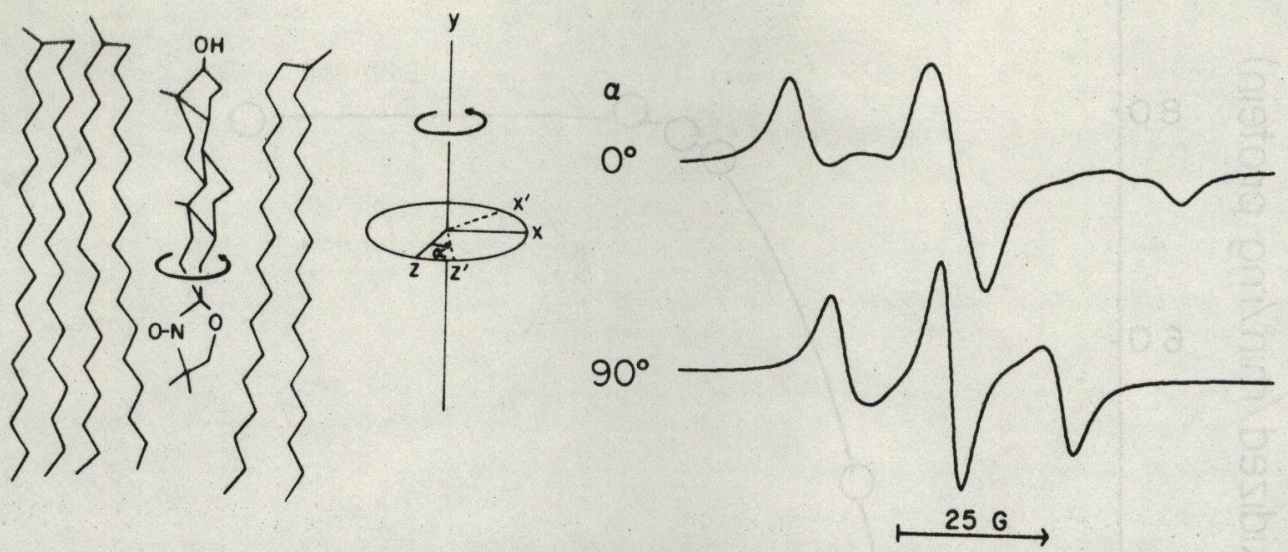

Fig. 7. Schematic drawing of a phospholipid bilayer region containing the spin label, 3-doxyl-5 $\alpha-$ androstan-17\%-o1. The principal degree of freedom is anisotropic motion about the $y$ axis of the $\mathrm{N}-\mathrm{O}$ moiety. On the right are spectra calculated for two extreme cases of no motion (top spectrum, $\alpha=0^{\circ}$ ) and large amplitude y axis molecular motion (bottom spectrum, $\alpha=90^{\circ}$ ).

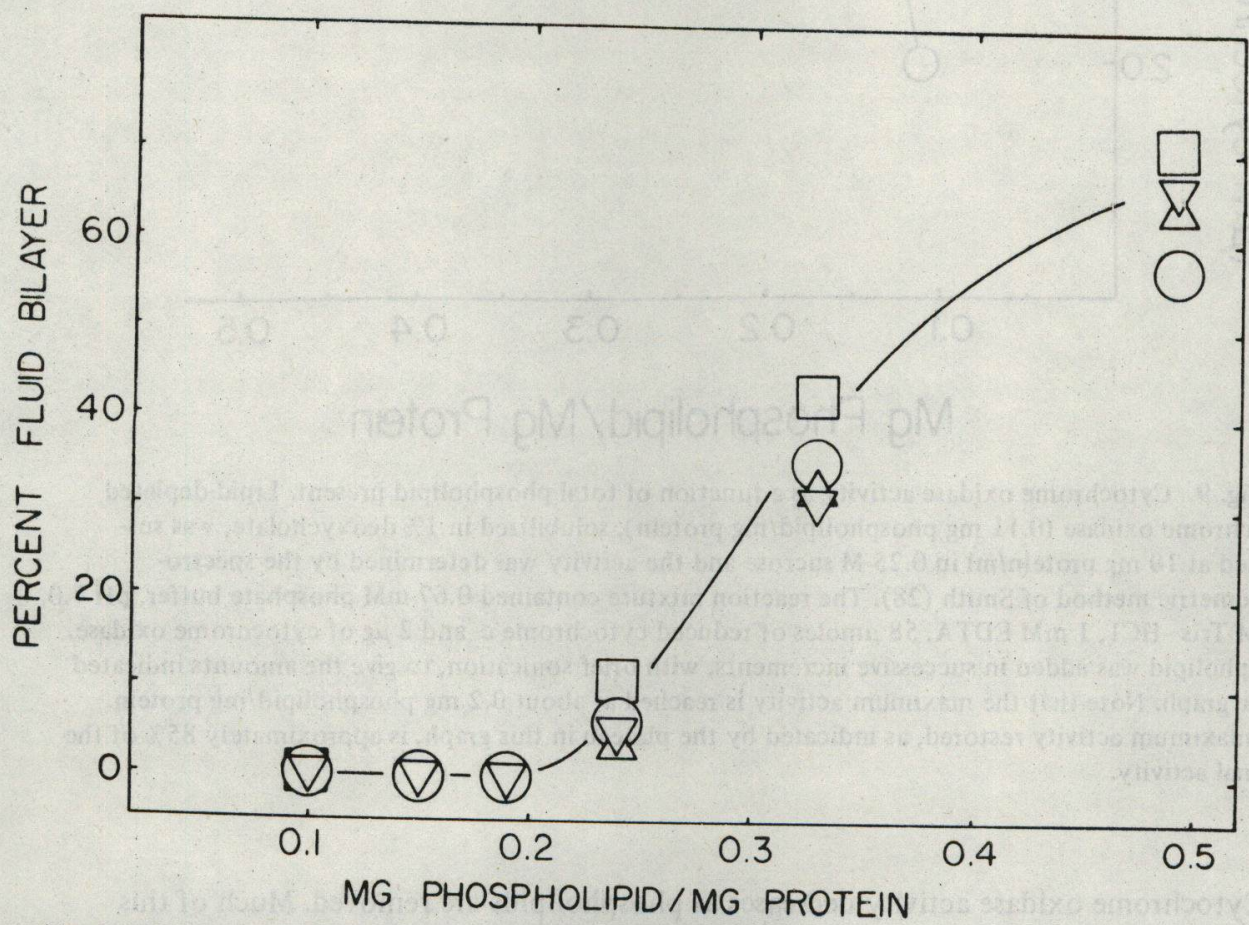

Fig. 8. Summary of calculations of percent fluid bilayer in cytochrome oxidase preparations, as a function of phospholipid content. Three calculations were performed using the 16 -doxylstearic acid $\nabla, \Delta, \circ)$, and the fourth calculation is based on data obtained with the 3 -doxyl-5 $\alpha$-androstan-17 $\beta$ ol spin label ( $\square$ ). Two estimations were based on summation of the fluid component (Fig. 3e) with either the spectrum from $0.10 \mathrm{mg}$ phospholipid $/ \mathrm{mg}$ protein $(\nabla)$ or the spectrum from $0.19 \mathrm{mg}$ phospholipid/ $\mathrm{mg}$ protein $(\Delta)$. The third estimate based on 16-doxylstearic acid data $(0)$ was obtained by subfrom each spectrum increments of a bound component $(0.10 \mathrm{mg}$ phospholipid $/ \mathrm{mg}$ protein, Fig. $3 \mathrm{a})$ from each spectrum of higher lipid content. A parallel subtraction method, using the steroid spin label, yielded the fourth set of points $(\square)$. This figure points out the substantial agreement between these estimates of the percent fluid bilayer. 


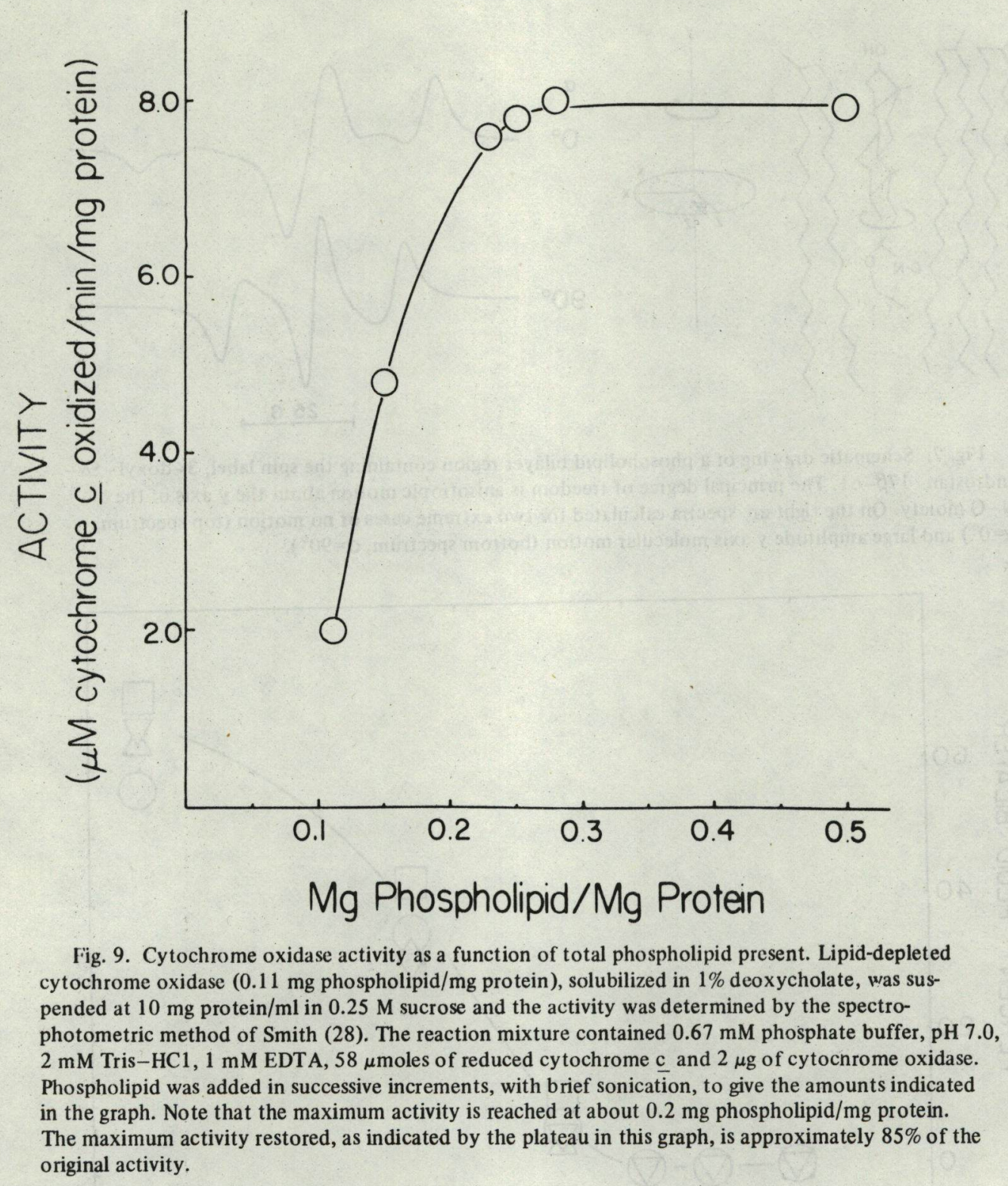

Cytochrome oxidase activity decreases as phospholipids are removed. Much of this activity can be restored by adding back phospholipids $(25-27)$. We have assayed preparations similar to those used in the spin labeling experiments and the results are shown in Fig. 9. The activity rises rapidly until the phospholipid reaches $0.2-0.25 \mathrm{mg}$ phospholipid/ $\mathrm{mg}$ protein, and then additional phospholipid has little effect on the cytochrome oxidase activity.

The bound lipid is a requirement for maximal cytochrome oxidase activity and apparently also plays a role in thermal stability of the enzyme complex (10). Thus, all of the available data suggest that $\sim 0.2 \mathrm{mg}$ phospholipid/mg protein is a "magic number" 


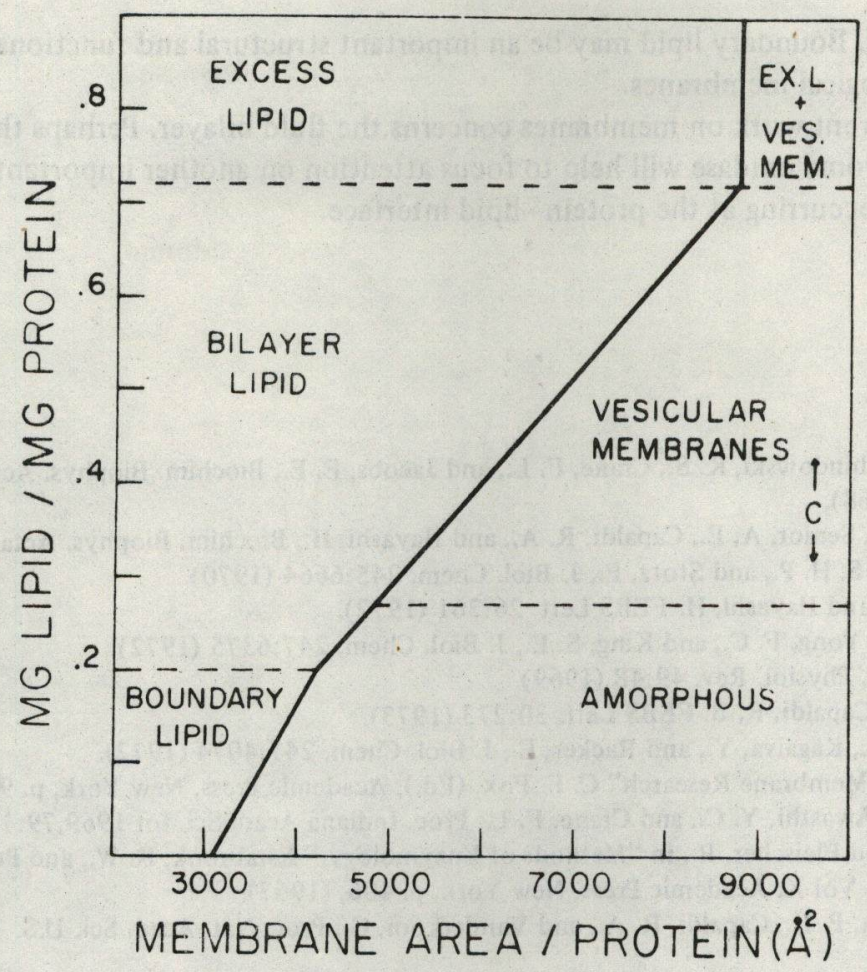

Fig. 10. Correlation of various phases observed in the phospholipid-cytochrome oxidase system. On the right, $\mathrm{C}$ designates the range of phospholipid concentrations where crystallinity (see Fig. 1b) may occur. The area of the protein is approximately $3100 \AA$.

for cytochrome oxidase. On the basis of the spin labeling data we interpret this number as representing boundary lipid, immobilized at the protein-lipid interface.

A general summary of the cytochrome oxidase system is shown in the phase diagram of Fig. 10. The relationship between the calculated membrane area/protein complex and the phospholipid content is given by the dark line. Below $0.2 \mathrm{mg}$ phospholipid $/ \mathrm{mg}$ protein all lipid is bound and the samples are amorphous. At this point there is a change in slope, reflecting the probable increased area per phospholipid molecule in the fluid bilayer. As the phospholipid content increases the percentage of fluid bilayer increases. For example, in typical cytochrome oxidase membranes with $0.4-0.5 \mathrm{mg}$ phospholipid/mg protein, approximately $30-40 \%$ of the membrane surface area is calculated to be occupied by fluid bilayer, with the protein complexes and their associated boundary lipid occupying the remainder of the surface area. Cytochrome oxidase membranes do not take up phospholipids indefinitely, and beyond about $0.7 \mathrm{mg}$ phospholipid/mg protein the excess phospholipid forms lipid vesicles $(10,13)$.

The boundary lipid concept appears to account for all of the available data on the cytochrome oxidase system. Since our experiments are limited to this system, we can only speculate on the generality of the model. We believe it likely, however, that other intrinsic membrane proteins bind lipid at the protein-lipid interface, and that this immobilized boundary lipid may play a role in conformational changes and stability of the 
membrane proteins. Boundary lipid may be an important structural and functional component in biological membranes.

Much of the current work on membranes concerns the fluid bilayer. Perhaps these findings on cytochrome oxidase will help to focus attention on another important lipid environment, that occurring at the protein-lipid interface.

\section{REFERENCES}

1. Sun, F. F., Prezbindowski, K. S., Crane, F. L., and Jacobs, E. E., Biochim. Biophys. Acta 153:804 (1968).

2. Vanderkooi, G., Senior, A. E., Capaldi, R. A., and Hayashi, H., Biochim. Biophys. Acîa 274:38 (1973).

3. Love, B., Chan, S. H. P., and Stotz, E., J. Biol. Chem. 245:6664 (1970).

4. Capaldi, R. A., and Hayashi, H. FEBS Lett. 26:261 (1972).

5. Kuboyama, M., Yong, F. C., and King, S. E., J. Biol. Chem. 247:6375 (1972).

6. Lemberg, M. R., Physiol. Rev. 49:48 (1969).

7. Komai, H. and Capaldi, R. B. FEBS Lett. 30:273 (1973).

8. Schneider, D. L., Kagaiva, Y., and Racker, E., J. Biol. Chem. 247:4074 (1972).

9. Racker, E., in "Membrane Research" C. F. Fox, (Ed.), Academic Press, New York, p. 97, (1972).

10. Chuang, T. F., Awasthi, Y. C., and Crane, F. L., Proc. Indiana Acad. Sci. for 1969,79:110 (1970).

11. Fleischer, S., and Fleischer, B., in "Methods of Enzymology," Estabrook, R. W., and Pullman. M. Z., (Eds.), Vol X, Academic Press, New York, p. 406, (1967).

12. Jost, P., Grifrith, P. H., Capaldi, R. A., and Vanderkooi, G., Proc. Nat. Acad. Sci. U.S. 70:480 (1973).

13. Jost, P., Griffith, O. H., Capaldi, R. A., and Vanderkooi, G., Biochim. Biophys. Acta (in press, 1973).

14. Griffith, O. H., Jost, P., Capaldi, R. A., Vanderkooi, G., Ann. N.Y. Acad. Sci. (in press, 1973).

15. Engelman, D. M., J. Mol. Biol. 58:153 (1971).

16. Benson, A. A., J. Amer. Oil Chemists' Soc. 43:265 (1966).

17. Awasthi, Y. C., Chuang, T. F., Keenan, T. W., and Crane, F. L., Biochim. Biophys. Acta 226:42 (1971).

18. Libertini, L. J., Waggoner, A. S., Jost, P. C., and Griffith, O. H., Proc. Natl. Acad. Sci. U.S. 64:13 (1969).

19. Jost, P., Libertini, L. J., Hebert, V. C., and Griffith, O. H., J. Mol. Biol. 59:77 (1971).

20. Hubbell, W. L., and McConnell, H. M., J. Amer. Chem. Soc. 93, 314 (1971).

21. Hubbell, W. L., and McConnell, H. M., Proc. Nat. Acad. Sci. U.S. 63:16 (1969).

22. Hsia, J. C., Schneider, H., and Smith, I. C. P., Biochim. Biophys. Acta 202:399 (1970).

23. Birrell, G. B., Van, S. P., and Griffith, O. H., J. Amer. Chem. Soc. 95:245 (1973).

24. Hubbell, W. L., and McConnell, H. M., Proc. Nat. Acad. Sci. U.S. 64:20 (1969).

25. Crane, F. L., Chuang, T. F., and Awasthi, Y. C., in "Current Trends in the Biochemistry of Lipids," Smellie, R. M. S. (Ed.), Academic Press, New York, p. 423, (1972).

26. Brierley, G. P., and Merola, A. J., Biochim. Biophys. Acta 64:205 (1962).

27. Fleischer, S., Brierley, G. P., Klouwen, H., and Slautterback, D., J. Biol. Chem. 237:3264 (1962).

28. Smith, L., in "Methods of Biochemical Analysis," Glick, D. (Ed.), Interscience, New York, Vol. II, p. 427 (1955). 